\title{
Empyema thoracis in an infant: A case report
}

\author{
V. Kotrashetti', Vijay Baburao Sonawane ${ }^{2}$, Kapil Bainade ${ }^{3}$, Shweta Nair ${ }^{4}$, Saili Bunde \\ From ${ }^{1}$ Professor, and Head of Unit, ${ }^{2}$ Associate Professor, ${ }^{3}$ Assistant Professor, ${ }^{4}$ Senior Resident, ${ }^{5}$ Junior Resident, Department of Pediatrics, D. Y. Patil \\ University, School of Medicine, Mumbai, Maharashtra, India
}

\begin{abstract}
Pneumonias are often associated with pleural effusions and a small proportion of them progress to empyema. Empyema thoracis is a complication of untreated or partially treated bacterial pneumonia. It is still responsible for significant morbidity and mortality. Due to poor facilities for culture, delay in seeking medical opinion, and indiscriminate use of antibiotics, it is very difficult to isolate microorganisms. The most common organism causing empyema in infants is Staphylococcus aureus. Cases of empyema thoracis by Methicillin-resistant S. aureus (MRSA) though rare are also been reported. We hereby report a case of empyema caused by MRSA. Baby responded well following chest tube drainage along with administration of antibiotics. The present case highlights the importance of early interventions by tube thoracostomy and appropriate antibiotics for better outcome. Imaging plays an important role in the diagnosis and management of empyema.
\end{abstract}

Key words: Empyema thoracis, Infant, MRSA, Tube thoracostomy, Pleural fluid

$\mathrm{P}$ ediatric empyema thoracis is a complication of bacterial pneumonia. Pulmonary pneumatoceles are thin-walled, air-filled cysts that develop within the lung parenchyma as a complication of pneumonia. They can also result from non-infectious etiologies such as trauma and positive pressure ventilation [1]. The prevalence of empyema is predominant in spite of advent newer antibiotics still resulting in significant morbidity and mortality. Most often, pneumatocele is commonly seen in infants and young children as a complication of Staphylococcus aureus pneumonia [2,3]. Detailed clinical history, physical examination, and relevant routine and specific investigation should be done. The pleural fluid should be studied for Gram staining, microscopy, cytology, pleural fluid culture, and antibiotic sensitive pattern. All the patients are to be treated with tube thoracostomy and antibiotic therapy depending on the culture and sensitivity pattern.

\section{CASE REPORT}

A 49-day-old baby was admitted to our hospital with a history of cough for 2 days, fever, vomiting, rapid breathing, and refusal to feed for 1 day. The child was born at 38 weeks of gestation and birth weight of $3.4 \mathrm{~kg}$ by lower segment cesarean section (in view of transverse lie). The baby did not cry immediately after

\section{Access this article online}

Received - 05 August 2020

Initial Review - 29 August 2020

Accepted - 15 October 2020

DOI: $10.32677 / \mathrm{IJCH} .2020 . v 07.110 .009$ birth and required bag and mask ventilation after which oxygen therapy was given for $24 \mathrm{~h}$. He had a history of early-onset neonatal sepsis. The patient also had convulsion on day 3 of life for which anticonvulsants were started. On day 6 , the patient was discharged. The child had a history of bottle feeding. On day 47 of life, the child had fever and cough for which the child was admitted and investigated in outside hospital. Total leukocyte count (TLC) and C-reactive protein (CRP) were raised. Chest $\mathrm{X}$-ray was normal (Fig. 1). The child had received injectable antibiotics for 1 day. Childs condition deteriorated hence the child was shifted to our hospital.

On examination, he was afebrile, tachycardia and tachypnea were present. Saturation on room air was $88 \%$. On respiratory system examination, trachea was shifted to right, air entry was reduced on the left side, dull note on left side and bilateral crepitations were present. Other systemic examination was normal. The child was not maintaining saturation on nasal prongs and distress was increasing, so the child was started on mechanical ventilation.

Blood investigations were done. Blood gas analysis showed metabolic acidosis. Complete blood count (CBC) showed low hemoglobin, increased TLC $\left(52,200 / \mathrm{mm}^{3}\right)$ with $92 \%$ polymorphs. CRP was elevated $(192 \mathrm{mg} / \mathrm{dl})$. Chest X-ray was done which was suggestive of left-sided effusion and pneumatoceles (Fig. 2) which gradually kept on increasing in size (Fig. 3). Highresolution computed tomography (HRCT) chest was suggestive of left-sided multiloculated draining empyema with atelectasis, consolidation, and pneumatoceles (Fig. 4). Intercostal drainage

Correspondence to: Dr. Saili Bunde, 801, Shree Sai Deep Apt, Near Bhagirathi, Bungalow, Bhusar Ali, Kalwa, Thane, West. E-mail: sailibunde28@gmail.com

(C) 2020 Creative Commons Attribution-NonCommercial 4.0 International License (CC BY-NC-ND 4.0). 


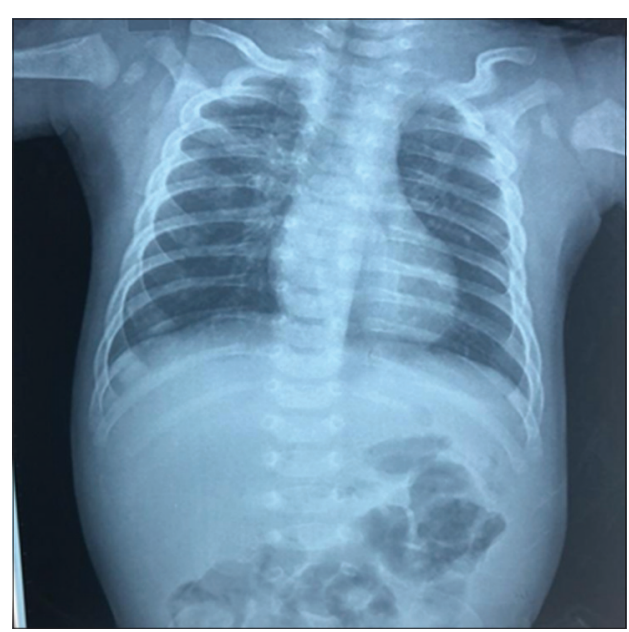

Figure 1: Normal chest X-ray on day 1 of illness

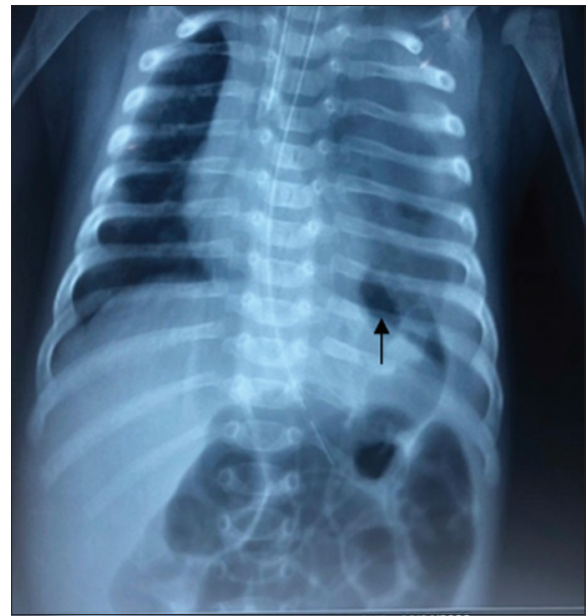

Figure 2: Left-sided effusion with pneumatocele

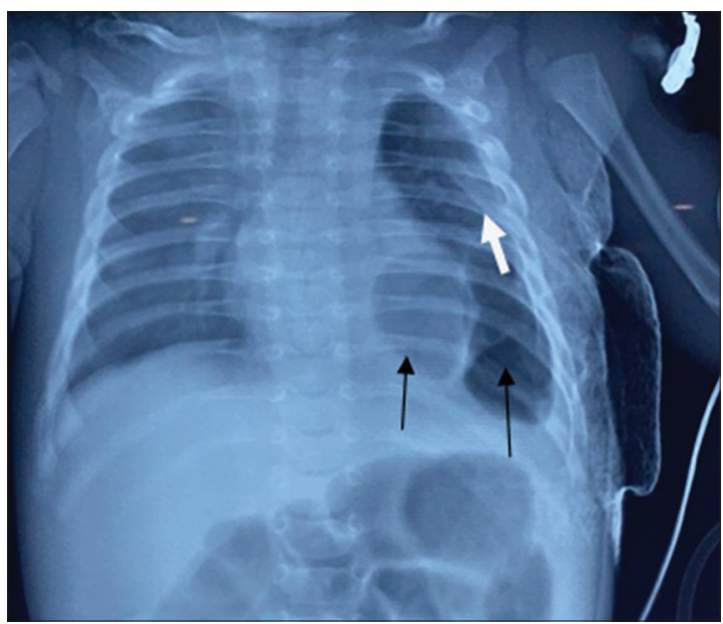

Figure 3: Pneumatocele increase in size (black arrow) reduced with ICD in situ (white arrow)

tube (ICD) was inserted (Fig. 5) and fluid was sent for examination which was suggestive of empyema (total protein $=3.7 \mathrm{~g} / \mathrm{dl}$, $\mathrm{TLC}=100$ cells $/ \mathrm{mm}^{3}$ with $70 \%$ polymorphonuclear cells, lactate dehydrogenase $=1597 \mathrm{IU} / \mathrm{L}$ ).

The child was treated with meropenem at $20 \mathrm{mg} / \mathrm{kg} / \mathrm{dose}$ and vancomycin at $15 \mathrm{mg} / \mathrm{kg} /$ dose, inotropes (dopamine and

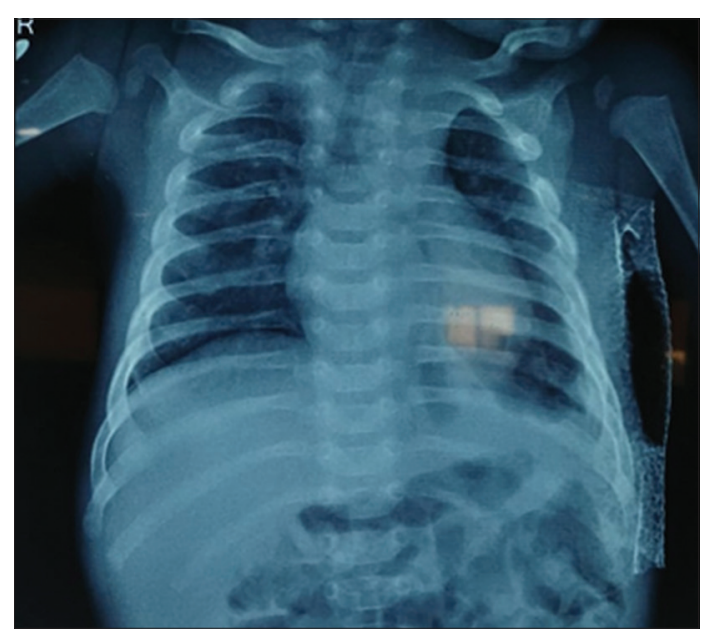

Figure 4: Pneumatocele and effusion

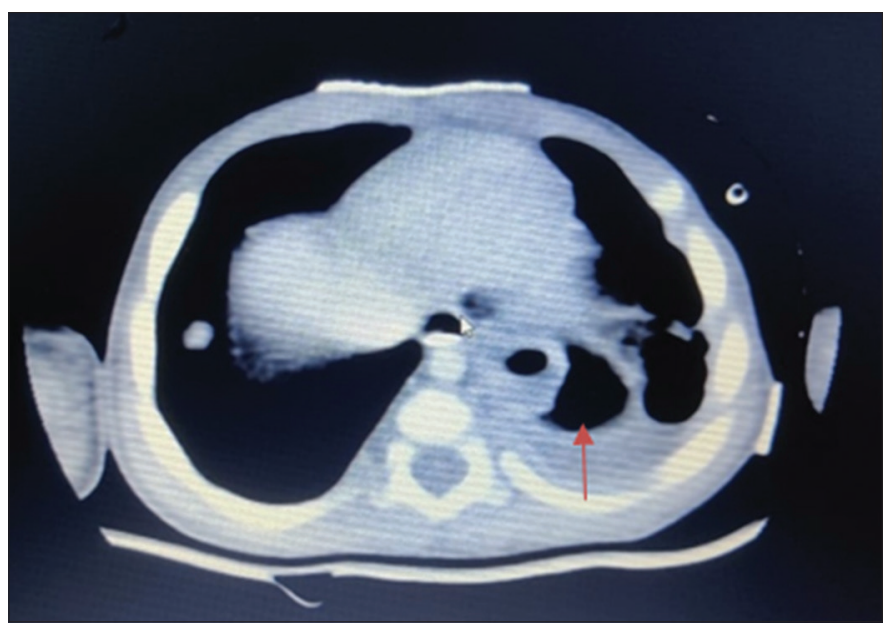

Figure 5: HRCT showing pneumatocele

dobutamine), packed red blood cell (PRBC) transfusion, and total parenteral nutrition (TPN). Gradually over 8 days, ventilatory settings were tapered and inotropes were omitted. On day 9, the patient was extubated. Chest physiotherapy and nebulization were given. Oral feeds were started and increased gradually. Pleural fluid and blood cultures were sterile. ET tube culture showed MRSA following which sensitive antibiotics were started. The patient improved clinically with antibiotic administration and physiotherapy. Air entry improved and pneumatoceles reduced on X-ray (Fig. 4). On day 19, ICD was removed. IV antibiotics were given for total 21 days and then shifted on oral antibiotics for 2 weeks. On follow-up visit, oral antibiotics were stopped after 2 weeks as per improvement. Since then, the patient is asymptomatic.

\section{DISCUSSION}

Empyema thoracis is defined as "pus in the chest" or presence of microorganism in the pleural fluid. Overall, $0.6 \%$ of childhood pneumonias are complicated by parapneumonic effusion which may progress to empyema thoracis. It predominantly involves in the right lung and $7.1 \%$ are bilateral [4].

Pneumonias in infancy can be a difficult clinical and radiographic diagnosis: An initial normal chest radiograph in 
infants does not eliminate the diagnosis of pneumonia like in our case where the $1^{\text {st }} \mathrm{X}$-ray was normal. For that reason, all cultures should be taken as soon as possible and the treatment initiated along with a continuous clinical evaluation of the infant for further investigations to detect complications such as pneumatoceles (repeat chest X-ray, redo blood test). A pulmonary pneumatocele is a frequent radiological finding that can occur during the course of acute pneumonia in children. Pneumatoceles are differentiated from lung abscesses, another radiological finding in the course of pneumonia, due to their tendency to rapidly change in appearance, size, and location [2]. The incidence of the formation of pneumatocele ranges from $2 \%$ to $8 \%$ of all cases of pneumonia in children with incidence up to $85 \%$ in $S$. aureus pneumonias [2,3].

Empyema may occur at any age, but at a very young and the debilitated elderly seem particularly susceptible. In the reports of Le Roux [5] and Benfield [6], one-half of all empyema's patients had complications of primary lung infection. Diagnostic aspiration of grossly purulent material from the involved pleural cavity established the diagnosis in all cases. In the pre-antibiotic era, empyema was a common complication of pneumonia, usually caused by pneumococcal and streptococcal organisms. However, with the control of these organisms by the use of the appropriate antibiotics, a whole new spectrum of infecting organisms, S. aureus, Escherichia coli, Klebsiella, Pseudomonas sp., Bacteroides sp., Proteus sp., Aerobacter aerogenes, and Streptococcus pyogenes, has emerged [7].

In 137 cases, the culture of the aspirates was positive for bacteria. Therapy before admission is probably largely responsible for the high percentage of sterile aspirates. Nearly all patients were exposed to preadmission antibiotic medication. Suboptimal courses of antibiotic modify the clinical course of the disease, resulting in inapparent or suppressed infection and the formation of sterile abscesses which was seen in our patient.

The work of Benfield [2] supports this observation; 38\% of his cases had sterile cultures, and $88 \%$ of the patients had antibiotics before admission. S. aureus was the most frequently recovered organism in the aspirates of the author's patients following pneumonia infection [7-9]. The conventional chest radiograph still provides the simplest and most accurate means of gaining diagnostic information regarding intrathoracic abnormalities. The chest X-ray films showed varying degrees of opacity of the involved hemithorax and shifting of the mediastinal structures in all patients. The chest radiograph was invaluable in the assessment of the progressive healing of the underlying pulmonary parenchyma pathology, the resolution of the effusion, and the determination of full expansion of the lung.
The mainstay in the treatment of empyema is the administration of sensitive antibiotics combined with the immediate institution of catheter drainage. The use of large-bore drainage catheters in the authors' series improved the prognosis of the patients [9]. To prevent pneumococcal diseases, the WHO recommended the inclusion of PCVs in childhood immunization programs worldwide [10].

\section{CONCLUSION}

Our case highlights the importance of maintaining a high index of suspicion of pneumonia in children even with normal radiological findings with clinical signs and symptoms of respiratory illness. The initiation of adequate antibiotics is essential to prevent further complications. Clinicians should search for pneumatoceles and other complications when the pneumonia is inadequately treated. Surgical approaches are indicated in the case when respiratory function is compromised or infected pneumatoceles and pneumothorax.

\section{REFERENCES}

1. Kesieme EB, Kesieme CN, Akpede GO, Okonta KE, Dongo AE, Gbolagade AM, et al. Tension pneumatocele due to enterobacter gergoviae pneumonia: A case report. Case Rep Med 2012;2012:808630.

2. Meyers HI, Jacobson G. Staphylococcal pneumonia in children and adults. Radiology 1959;72:665-71.

3. Ceruti E, Contreras J, Neira M. Staphylococcal pneumonia in childhood. Long-term follow-up including pulmonary function studies. Am J Dis Child 1971;122:386-92.

4. Kuti BP, Oyelami OA. Risk factors for parapneumonic effusions among children admitted with community-acquired pneumonia at a tertiary hospital in South-West Nigeria. Afr J Respir Med 2014;10:26-34.

5. LeRoux BT. Empyema thoracis. Br J Surg 1965;52:89-99.

6. Benfield GF. Recent trends in empyema thoracis. $\mathrm{Br} \mathrm{J}$ Dis Chest 1981;75:358-66.

7. Geha AS. Pleural empyema. Changing etiologic, bacteriologic, and therapeutic aspects. J Thorac Cardiovasc Surg 1971;61:626-35.

8. Aderele WI, Stevenson CE, Antia AU. Empyema in childhood: Review of 116 cases. Niger J Paediatr 1974;1:20-5.

9. Stiles QR, Lindesmith GC, Tucker BL, Meyer BW, Jones JC. Pleural empyema in children. Ann Thorac Surg 1970;10:37-44.

10. World Health Organization. Pneumococcal conjugate vaccine for childhood immunization--WHO position paper. Wkly Epidemiol Rec 2007;82:93-104.

Funding: None; Conflicts of Interest: None Stated.

How to cite this article: Kotrashetti V, Sonawane VB, Bainade K, Nair S, Bunde S. Empyema thoracis in an infant: A case report. Indian J Child Health. 2020; 7(10):427-429. 